\title{
Design and Analysis of Test Signals for System Identification
}

\author{
LIU Bo, ZHAO Jun, and QIAN Jixin \\ Institute of System Engineering \\ National Key Laboratory of Industrial Control Technology \\ Zhejiang University, Hangzhou, P.R. China 310027 \\ liubo@iipc.zju.edu.cn
}

\begin{abstract}
For multi-channel process, due to disadvantages of the open-loop single variable step method, multi-channel test method is used. That means all of the channels are tested at the same time. In order to eliminate cross-effect of the different test signals, it requires that all the test signals are uncorrelated. Several test signals are introduced and analyzed. Based on two familiar identification methods: correlation analysis method and least-squares method, we put our strength on the way to get uncorrelated test signals. A novel design for the period length of uncorrelated pseudo random binary sequence (PRBS) is proposed. Use this design method, identifiable PRBS signals can be gained and their periods are the shortest. Simulation results show the effectiveness.
\end{abstract}

\section{Introduction}

The fundamental problem in all kinds of predictive control is how to choose an appropriate functional model which reflects the characteristic of the control system. Again, The primary method to acquire a valuable functional model in the current industry is system identification. And the key to obtain a worthful result of correct identification is choosing rightly recognizable test signals.

Although multivariable control concept is well accept by industry, the guideline for identification practice is still single variable thinking. Current practice of industry is to use a series of single variable step tests ${ }^{[1]}$ for model identification, which reflects the fact that each manipulating variable (MV) is stepped separately and some clear step responses are expected for modeling each transfer function. The biggest problem of this test method is its high cost in time and manpower. The product qualities may be disturbed by stepping the MVs. This problem can be solved by using automatic multivariable test approach. But when you input several test signals at the same time, there are some cross-effect between the test signals. For the sake of identifying different impacts on the same output from different inputs in such experiment, these inputs need to be presumed as uncorrelated. Therefore, for multivariable process, we have new design for uncorrelated test signals. Simulation data show that use these design methods, less test time needs and better results can be get. 


\section{Pseudo Random Binary Sequence (PRBS)}

PRBS is a long period signal. In a period, it is a true stochastic binary signal. Over a period, it is a deterministic signal and it will repeat the previous period signal. In the field of system identification, the stochastic of PRBS plays a quite important role.

\subsection{Properties of PRBS}

A PRBS is a two states signal which can be generated by using a feedback shift register. When the number of registers are $\mathrm{n}$, the maximum possible length is : $N=2^{n}-1$. Assume that the clock period is $\Delta t$, the period of PRBS is: $T=N \cdot \Delta t$.

Because the length of PRBS is odd number, in a period, it takes two values -a and $a$, the number of value " $+a$ " is one less than the number of value" $-a$ ".

The most characteristic of PRBS signal is its auto-correlation function can resemble the auto-correlation of white noise sequence. For this characteristic, it can be used as test signal. Its auto-correlation function is:

$$
R_{x x}(\tau)=\left\{\begin{array}{cc}
a^{2}\left(1-\frac{N+1}{N} \cdot \frac{|\tau|}{\Delta t}\right) & -\Delta t<\tau<\Delta t \\
-\frac{a^{2}}{N} & \Delta t \leq \tau \leq(N-1) \Delta t
\end{array}\right.
$$

That is, when $\mathrm{N}$ is large enough, in the range $[0, N \Delta t], R_{x x}(\tau)$ can be taken as $\delta$ function. See Fig 1.

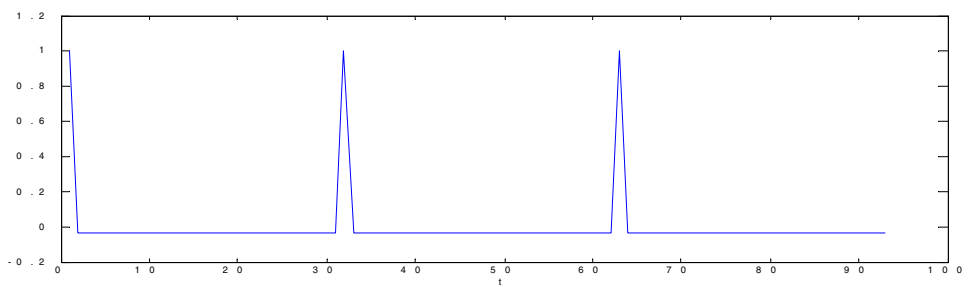

Fig. 1. Auto-correlation function of PRBS

Note that generating a part of a period of a PRBS will not give a signal with the above properties. This makes the selection of time less flexible.

\subsection{PRBS Used in Correlation Analysis Method}

Firstly for SISO linear system, suppose the input of linear system is $x(t)$, output is $y(t)$. Correlation analysis method uses correlation method to solve the dynamic characteristic of system, that is impulse response $g(\tau)$. The cross-correlation function between input and output is 


$$
R_{x y}(\tau)=\int_{0}^{T_{s}} g(s) R_{x x}(\tau-s) d s
$$

Where, $T_{s}$ is system adjust time, when $\tau \geq T_{s}$, we get $g(\tau)=0$. Adjust the period of PRBS, it can be arrived that $T>T_{s}$.

According to (3), we have

$$
g(\tau)=\left\{\begin{array}{cc}
{\left[R_{x y}(\tau)-R_{y x}(\tau)\right] / S} & \tau \geq \Delta t \\
{\left[R_{x y}(\tau)-R_{y x}(\tau)\right] /\left[\left(\frac{1}{2}+\frac{\tau}{\Delta t}\left(1-\frac{\tau}{2 \Delta t}\right)\right) S\right]} & \Delta t>\tau>0 \\
2\left[R_{x y}(0)-R_{y x}(\Delta t)\right] / S & \tau=0
\end{array}\right.
$$

where

$$
S=\left(1+\frac{1}{N}\right) a^{2} \Delta t
$$

Consequently, we can achieve $g(\tau)$ from cross-correlation function between input and output conveniently taking PRBS as input signals. In relative analysis method, used as test signal and in virtue of its periodicity, PRBS does not need infinite time to calculate cross-correlation function in contrast with white noise sequence. PRBS just needs a period time. Therefore to ensure yielding fine identification precision, PRBS shall be easily made and save more time.

As to MIMO system, an old approach is to test every variable independently. Assuming other inputs are zero, we test one selected variable at each time. This approach is time-consuming for one by one test method. Again, it doesn't guarantee to keep other variables intact when checking the selected one. To solve this problem, it needs to make a one and only experiment. But when you input several test signals at the same time, there are some cross-effect between the test signals, that it's difficult to distinguish different impacts on the same output from different inputs. For the sake of identifying different impacts on the same output from different inputs in such experiment, these inputs need to be presumed as uncorrelated.

In order to make uncorrelated PRBS signals, firstly you need to generate a PRBS, secondly shift it horizontally. That is to say, other signals will be this signal's delaysignals. But it still exits a big problem that what is the delay value. To guarantee process dynamic is persistent excited by input signals, the PBRS signal must ensure that its period is longer than the settling time of process for each variable. For this reason, the uncorrelated range of each variable should be longer than its settling time. Therefore, in order to ensure the PRBS signals are uncorrelated and identifiable, what is the minimum delay value we should choose? Aim at this problem we have further study about delay value $D$.

Theorem 1. Assume a linear process with m-input and n-output, where $n \leq m$, $x_{i}(t)(i=1, \cdots, m)$ are input PRBS signals, $x_{i+1}(t)$ are delay signals of $x_{i}(t)$, suppose the delay value are $K_{i+1} \Delta t$, that is $x_{i+1}(t)=x_{i}\left(t+K_{i+1} \Delta t\right)$. All the input signals are uncorrelated in the range of $0 \sim\left(N-\sum_{i=1}^{m} K_{i}-1\right) \Delta t$, where $K_{1}=0$ is the delay value of $x_{1}(t)$. 
Proof. Consider $x_{1}(t)$ is a PRBS signal, $x_{2}(t)$ is the delay signal of $x_{1}(t)$, the delay value is $K_{2} \Delta t$, so that $x_{2}(t)=x_{1}\left(t+K_{2} \Delta t\right)$.

The cross-correlation function between $x_{1}(t)$ and $x_{2}(t)$ is

$$
\begin{aligned}
R_{x_{1} x_{2}}(\tau)=\frac{1}{T} \int_{0}^{T} x_{1}(t) x_{2}(t+\tau) d t & =\frac{1}{T} \int_{0}^{T} x_{1}(t) x_{1}\left(t+\tau+K_{2} \Delta t\right) d t \\
=R_{x_{1} x_{2}}\left(\tau+K_{2} \Delta t\right) & 0<\tau<\left(N-K_{2}-1\right) \Delta t
\end{aligned}
$$

According to (5), the cross-correlation function between $x_{i}(t)$ and $x_{i-p}(t)$ is

$$
\begin{aligned}
R_{x_{i} x_{i-p}}(\tau) & =\frac{1}{T} \int_{0}^{T} x_{i}(t) x_{i-p}(t+\tau) d t \\
& =\frac{1}{T} \int_{0}^{T} x_{i-p}(t) x_{i}\left(t+\tau+\left(K_{i}+K_{i-1}+\cdots+K_{i-p+1}\right) \Delta t\right) d t \\
& =R_{x_{i-p} x_{i-p}}\left(\tau+\left(K_{i}+K_{i-1}+\cdots+K_{i-p+1}\right) \Delta t\right) \\
& =\frac{-a^{2}}{N} \quad 0<\tau \leq\left(N^{\prime}-\left(K_{i}+K_{i-1}+\cdots+K_{i-p+1}\right)-1\right) \Delta t
\end{aligned}
$$

when $\mathrm{N}$ is large enough, $R_{x_{i} x_{i-p}}(\tau) \approx 0$, so $x_{i}(t)$ and $x_{i-p}(t)$ will be uncorrelated in the range of $0 \sim\left(N-\left(K_{i}+K_{i-1}+\cdots+K_{i-p+1}\right)-1\right) \Delta t$. Thus it can be seen, the uncorrelated range of all signals is $0 \sim\left(N-\sum_{i=1}^{m} K_{i}-1\right) \Delta t$.

The theorem shows the uncorrelated range. Through this theorem, it can be known that which is the minimum delay value and we got a new way to design test signals. Consider a linear process with $\mathrm{m}$ inputs and $\mathrm{n}$ outputs, where $n \leq m$, assume that $D_{i}$ stands for the settling time for $i$ th input. So that the new design approach of test signals is:

\section{Step1: Generate a PRBS $x_{1}(t)$;}

Step2: Calculate $\max D_{i}(i=1, \cdots, m)$, choose the channel which has the maximum settling time as the first channel and take $x_{1}(t)$ as its input signal;

Step3: Choose the remaining input signals for $m-1$ channels stochastically $\left[\begin{array}{lllll}x_{2}(t) & x_{3}(t) & \cdots & x_{m-1}(t) & x_{m}(t)\end{array}\right] ;$

Step4: $\quad x_{i}(t)$ is the delay signal of $x_{i-1}(t)$, its delay value is $K_{i} \Delta t=D_{i}(i=1, \cdots, m)$, where $K_{1}=0$.

From the forward approach, the minimum period of input signals is $T=\sum_{i=1}^{m} D_{i}$. In reference [4], the author used maximum settling time of the process as delay value. So its period is $m \times D_{\max }$. By comparing $\mathrm{T}$ with $m \times D_{\max }$, especially to a quite complicated 
system in which every channel differs in the settling time, it bigly shortens the length of test signal after we make the improvement. The data of example 1 shows it clearly.

\section{Generalized Binary Noise (GBN)}

This signal was proposed by Tullenken. The motivation was to generate a test signal that is suitable for control-relevant identification of industrial processes.

\subsection{Properties of GBN}

A GBN signal $u(t)$ takes two values $-a$ and $a$. At each candidate switching time $\mathrm{t}$, it switches according to the following rule

$$
\begin{gathered}
P[u(t)=-u(t-1)]=p_{s w} \\
P[u(t)=u(t-1)]=1-p_{s w}
\end{gathered}
$$

where $p_{s w}$ is switching probability. The distribution for the event at each switching time is an independent alternative distribution with parameter $p_{s w}$.

The GBN auto-correlation function is

$$
\begin{aligned}
R_{u u}(\tau)= & E u(t) u(t-\tau) \\
= & \frac{1}{N} \sum_{t=1}^{N} a^{2}[P(u(t)=a \text { and } u(t-\tau)=a)+P(u(t)=-a \text { and } u(t-\tau)=-a) \\
& \quad-P(u(t)=a \text { and } u(t-\tau)=-a)+P(u(t)=-a \text { and } u(t-\tau)=a)] \\
= & \frac{1}{N} \sum_{t=1}^{N} a^{2}\left(1-2 p_{s w}\right)[P(u(t-1)=a \text { and } u(t-\tau)=a)+P(u(t-1)=-a \text { and } u(t-\tau)=-a) \\
& \quad-P(u(t-1)=a \text { and } u(t-\tau)=-a)+P(u(t-1)=-a \text { and } u(t-\tau)=a)] \\
= & \left(\left(1-2 p_{s w}\right) E(u(t-1) u(t-\tau))\right. \\
= & \left(\left(1-2 p_{s w}\right)^{\tau} E u^{2}(t-\tau)\right. \\
= & \left(1-2 p_{s w}\right)^{\tau} \frac{1}{N} \sum_{t=1}^{N} a^{2}(P(u(t-\tau)=a+P(u(t-\tau)=-a) \\
= & a^{2}\left(1-2 p_{s w}\right)^{\tau}
\end{aligned}
$$

Especially when: $p_{s w}=1 / 2$, then $1-2 p_{s w}=0$. Its auto-correlation function is

$$
R_{u u}(\tau)=\left\{\begin{array}{cc}
a^{2} & \tau=0 \\
0 & \tau \neq 0
\end{array}\right.
$$

From this function it can be seen that GBN auto-correlation function can resemble the auto-correlation function of white noise sequence. And especially when $p_{s w}=1 / 2$, the auto-correlation is the same with the auto-correlation of white noise sequence. It can be called white noise GBN. Shows in Fig 2. 


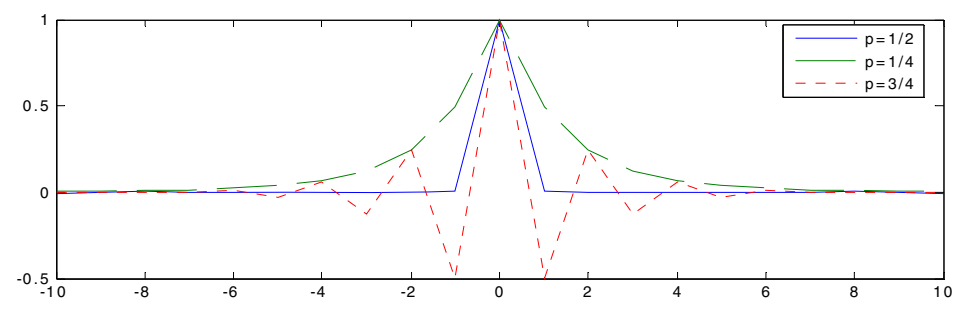

Fig. 2. Auto-correlation function of GBN for different $p_{s w}$

White noise GBN can be used in relative analysis method. Because its autocorrelation function is $\delta$ function, the cross-correlation between any of two white noise GBN signals is zero, that is to say any of two white noise GBN signals are uncorrelated. That is, for MIMO system, we can generate $\mathrm{m}$ white noise GBN signals as input signals.

Note that GBN noise has no period, it needs infinite time to calculate crosscorrelation function, which needs quite a long time for GBN to attain good precision. Compare with PRBS, PRBS is period signal, it just needs a period time to calculate its auto-correlation function. Therefore, PRBS shall be a better choice to be applied in relative analysis method.

\subsection{GBN Used in Least-Squares Method}

In least-squares method, to guarantee that the estimation algorithms have unique solutions, test signals should be persistent excitation. PRBS, L signal and GBN can be used in least-squares method.

In a process control environment, a PRBS signal will over-emphasize the high frequency band at the cost of the low and middle frequency band; its frequent fluctuations can be harmful for control actuators. And its power spectrum is

$$
\Phi(\omega)=\frac{2 \pi a^{2}}{N} \sum_{k=1}^{N} \delta(\omega-2 \pi k / N) \quad 0 \leq \omega \leq 2 \pi
$$

Its spectrum has dips around frequencies $2 \pi / \Delta t, 4 \pi / \Delta t, 6 \pi / \Delta t, \cdots$, which will result in low signal-to-noise ratios in these frequency ranges. A PRBS signal is not the best test signal for least-squares method.

A good test signal for process control has a low-pass character. We can generated low-pass GBN signals by reducing the switching probability $p_{s w}$, or equivalently, increasing the mean switching time $E T_{s w}$. Define minimum switching time $T_{\min }$ as the time (in samples) to keep the signal constant and switching time $T_{s w}$ as the elapsed time between two switches, we can get GBN power spectrum:

$$
\Phi_{u}(\omega)=\frac{\left(1-q^{2}\right) T_{\min }}{1-2 q \cos T_{\min } \omega+q^{2}}
$$

Note that unlike the PRBS signal, the spectrum of a low-pass GBN signal does not have dips at frequencies $2 \pi / \Delta t, 4 \pi / \Delta t, \cdots$, which is advantageous, see Fig 3 . So GBN signal is better than PRBS signal in least-squares method. 


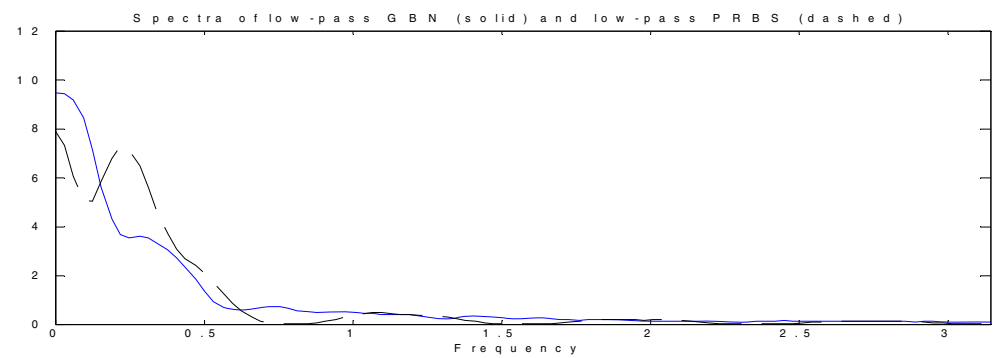

Fig. 3. Compare the power spectra of low-pass GBN and low-pass PRBS

\section{Examples}

Example 1. Consider a process with three inputs. The transfer functions are:

$$
G(s)=\left[\frac{1}{100 s+1} \frac{1}{20 s+1} \frac{1}{s+1}\right]^{T}
$$

Use correlation analysis method to identify this process. PRBS signals are taken as test signals. Follow the new design steps of PRBS, we get the period of PRBS. Table 1 shows the different period time by using delay value proposed in reference [4] and this paper.

Table 1. Compare the period of PRBS

\begin{tabular}{|c|c|c|c|}
\hline Process & Settling time & $\begin{array}{c}\text { The period of PRBS using } \\
\text { the method in reference [4] }\end{array}$ & $\begin{array}{c}\text { The period of PRBS using } \\
\text { the method of this paper }\end{array}$ \\
\hline Variable 1 & 500 & 1500 & 605 \\
\hline Variable 2 & 100 & 1500 & 605 \\
\hline Variable 3 & 5 & 1500 & 605 \\
\hline The whole process & & 1500 & 605 \\
\hline
\end{tabular}

Example 2. Let us see a typical petrochemical processes in refinery industry. There are four main variables. Asymptotic method ${ }^{[7]}$ extended by least-square method is used here. Choose white noise GBN signals as test signals. Because the uncorrelated character of white noise GBN, we can generate four white noise GBN signals directly and input to the process at the same time. Table 2 shows the duration of one test by using single variable step test and white noise GBN signals.

In multivariable test, as the four variables are test at the same time, the duration time of every variable are the same. Compared to the conventional single variable step test, our design can save about $70 \%$ test time. In such a petrochemical process, we can save most cost of time and money. 
Table 2. Compare the duration of one test

\begin{tabular}{|c|c|c|c|}
\hline Process & Settling time & $\begin{array}{c}\text { The duration of single } \\
\text { variable step test }\end{array}$ & $\begin{array}{c}\text { The duration using } \\
\text { multivariable test }\end{array}$ \\
\hline Main fractionator & 50 & 50 & 50 \\
\hline Atmospheric tower & 40 & 40 & 50 \\
\hline FCC react & 20 & 20 & 50 \\
\hline Stabilizer & 35 & 35 & 50 \\
\hline The whole process & & $>145$ & 50 \\
\hline
\end{tabular}

\section{Conclusions}

The result of system identification is affected by test signals directly. This article study several kinds of test signals from the point of view of system identification. Firstly, introduce every kind of test signal's attribute. Then analyse how to design uncorrelated test signals in correlation analysis method and least-squares method. In conclusion, due to the periodicity of PRBS, the time to calculate auto-correlation function is comparatively short. Therefore, PRBS shall be a better choice to be applied in relative analysis method than GBN. And we can obtain low-pass signal by converting the convert probability of GBN, such characteristics make GBN works better than PRBS in least-squares method. The future researches will focus more on these test signals more suitable for system identification.

\section{References}

[1] Chongzhi Fang, Deyun Xiao. Process Identification. Qinghua Publishing House. 1988.

[2] Ljung, L. System Identification: Theory for the User. NJ, Prentice-Hall, UpperSaddle River, 2nd edition, 1999

[3] M. leskens, L.B.M. Van kessel. MIMO closed-loop identification of an MSW incinerator. Control Engineering Practice, 2002, 10:315-326.

[4] M.E.H. Amrani, R.M. Dowdeswell, P.A. Payne, etal. Pseudo-random binary sequence interrogation technique for gas sensors[ J] . Sensors and Actuators, 1998, B 47:118-124

[5] Ljung, L. Asymptotic variance expressions for identified black-box transfer function models. IEEE Transactions on Automatic Control, 1985b, 30:834-844.

[6] Zhu, Y.C. Black-box identification of MIMO transfer functions: asymptotic properties of prediction error models. Int.J.Adaptive Control and Signal Processing, 1989, 3:357-373.

[7] Zhu, Y.C. Multivariable process identification for MPC: the asymptotic method and its applications. Journal of Process Control, 1998, 8(2):101-115.

[8] Bainan Li. PRBS signal and relative identification. Science Publishing House. 1987. 